\begin{tabular}{lllllllllllllllllllllllllllllllll}
\hline$R$ & $E$ & $V$ & I & S & T & A & D & E & E & S & T & U & D & I & O & S & I & N & T & E & $R$ & R & N & A & C & I & O & N & A & L & E & S
\end{tabular}

\title{
El Tratado de Libre Comercio entre Chile y Estados Unidos
}

\author{
Joseph Ramos \\ Alfie Ulloa Urrutia
}

El 6 de junio del 2003 Chile y Estados Unidos firmaron un Tratado que liberaliza plenamente el comercio de bienes y establece amplios compromisos en otras materias. Este logro no es aislado y debe considerarse como la consagración de una política chilena basada en el libre comercio y su solidez institucional. Se analizan algunas consideraciones teóricas respecto a los beneficios de la apertura con aplicaciones a Chile. Algunos comentarios respecto de ciertos capítulos del Acuerdo cierran el artículo.

\section{INTRODUCCIÓN}

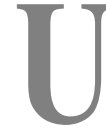

n Tratado de Libre Comercio (TLC) podría establecerse mediante un simple: "Las Partes en este Acuerdo establecen un área de libre comercio", y de hecho es lo que dice el primer Artículo del capítulo inicial del Tratado, al señalar que "las Partes" son Chile y Estados Unidos. Poco habría que agregar, de no ser porque al primer capítulo le siguen otros veintitrés, de una a cuarenta páginas y con decenas de artículos que li- mitan cuán libre será el comercio en esta área que ahora acuerdan.

En efecto, los acuerdos de libre comercio son complejos cuerpos legales que establecen las doctrinas, obligaciones y excepciones que regirán la relación comercial entre los socios, y mucho distan del utópico libre comercio de los modelos canónicos. Este trabajo empleará el texto final de las negociaciones que el pasado mes de diciembre concluyeron Chile y Estados Unidos ${ }^{1}$, y que fue firmado

NotA: Las opiniones aquí expresadas representan únicamente las de los autores y en ningún caso a la Universidad de Chile u otras dependencias en que se desempeñan. Los errores u omisiones son exclusiva responsabilidad de los autores.

${ }^{1}$ Disponible en www.ustr.gov. 
el día 6 de junio para someterse luego a la aprobación parlamentaria respectiva.

La estructura del capítulo es la siguiente: la Introducción, una cronología de los 12 años transcurridos entre el primer anuncio y la última ronda negociadora del Tratado; una reseña teórica de los efectos del libre comercio y el beneficio esperado para Chile; una síntesis de los capítulos más relevantes del Tratado y las conclusiones.

\section{UNA NEGOCIACIÓN CON HISTORIA}

Chile fue el primero en reaccionar a la "Iniciativa para las Américas" del presidente George Bush padre, y en su visita oficial a Chile en 1991 ya se hablaba de un Acuerdo ${ }^{2}$. Sin embargo, pasaron doce años y tres gobiernos antes de poder concretarlo, si se considera que ese mismo año ya se reunían los expertos para explorar el camino que culminó en diciembre de 2002 .

Tres años después, bajo la primera presidencia Clinton, la entrada en vigor del Tratado de Libre Comercio de América del Norte (TLCAN o NAFTA en su sigla inglesa) entre Estados Unidos, Canadá y México, marcaba el primer paso de esa agenda liberalizadora, que se consolidó a fines de año en la Primera Cumbre de las Américas con el "Pacto para el Desarrollo y la Prosperidad, Democracia,
Libre Comercio y Desarrollo Sustentable en las Américas". El medio para conseguirlo sería el "Acuerdo de Libre Comercio de las Américas" (ALCA) que conduciría el 2005 a un área de libre comercio entre los 34 países democráticos del hemisferio.

\section{Chile fue el primer país en reaccionar a la iniciativa estadounidense.}

En la Cumbre se anuncia también la ampliación del Nafta. Doce meses han pasado desde la entrada en vigor del tratado tripartito y se plantea la posibilidad de ampliarlo incorporando a Chile como primer socio invitado, "reconociendo" su desempeño económico y su vocación aperturista. Por entonces -hoy ya se olvidó- Nafta incluía una promesa de "ingreso" emulando el Acuerdo de Maastricht, si los aspirantes cumplían una lista calificadora. La noticia revolucionó a las autoridades de la época, la expectativa de incluirse a un bloque que agrupaba $400 \mathrm{mi}$ llones de consumidores movilizó los esfuerzos públicos y privados en la prioridad de política exterior del país.

Por desgracia para Chile, la ampliación del Nafta requería que el Congreso estadounidense le otorgara la facultad correspondiente al Ejecutivo para negociar nuevos tratados: "la vía rápida" o the Fast

${ }^{2}$ La "Iniciativa" se anunció en 1990, luego del ALC Estados Unidos-Canadá (1989) y anticipando lo que sería la política norteamericana en la era post desarticulación soviética y consolidación europea. Era una agenda de desarrollo que incluía aspectos económicos, comerciales y políticos, enfatizando paz, democracia y prosperidad como objetivo regional. Una actualización de la Doctrina Monroe de "América para los americanos" en un amplio sentido político-económico. 
Track trade negotiating authority, que se conocería luego como Trade Promotion Authority (TPA). Dicha ley especifica los socios, acota las materias y define los objetivos negociadores estadounidenses, a cambio de garantizar un proceso parlamentario expedito de autorización o rechazo, sin debate ni modificaciones y en un plazo determinado, a los acuerdos comerciales del Ejecutivo. Sin embargo, la apertura comercial es de los temas más controversiales en Estados Unidos y tras firmar el Nafta y la Ronda Uruguay de la Organización Mundial de Comercio (OMC), el Congreso negó durante ocho años la autorización al presidente para negociar nuevos acuerdos comerciales ${ }^{3}$.

La Segunda Cumbre de las Américas se realizó en Santiago de Chile en 1998. Para los anfitriones el Tratado con Estados Unidos seguía siendo prioritario, pero llegaban a esta reunión tras un largo camino recorrido en siete años. Chile firmaba en la Cumbre el acuerdo de libre comercio con México y cumplía diez meses de vigencia otro con Canadá, abarcando así a los socios del Nafta que no requerían Fast Track; había negociado parcialmente con todos sus vecinos, estaba asociado al Mercosur y anunciaba el inicio de negociaciones con Centroamérica para un acuerdo de libre comercio, en la misma cumbre comenzó la negocia- ción del ALCA. El Cuadro $\mathrm{N}^{\circ} 1$ (ver Anexos) sintetiza este impresionante proceso de apertura chileno.

\section{El país realizó un impresionante proceso de apertura.}

Clinton, en cambio, llega derrotado a la Cumbre de Santiago. El Congreso le ha negado otra vez el permiso para negociar acuerdos comerciales. Reconociendo este fracaso interno en convencer a sus congresistas de otorgarle el esquivo Fast Track $^{4}$, propone a Chile crear una Comisión de Comercio e Inversión es que adelante temas de la agenda bilateral con miras a un eventual Tratado. La Comisión efectuó varias reuniones, la última en octubre del 2000.

En noviembre del 2000, a pocos días de la Cumbre de Florianópolis, donde los países del Mercosur y Chile discutirían una estrategia común para enfrentar a Estados Unidos en el ALCA, llegó el anuncio formal del (re)inicio de las negociaciones para un Tratado de Libre Comercio entre Chile y Estados Unidos. Pocos meses faltan para que Clinton abandone la Casa Blanca y pocos han pasado desde la llegada de Lagos a La Moneda. Como acto simbólico para con la administración saliente, la primera ronda de

${ }^{3}$ La autorización que Clinton recibió en su primer mandato y derivó en la Ronda Uruguay, NAFTA, ALCA y APEC, expiró en junio del 1994 y sólo sería entregada nuevamente a George W. Bush en agosto del 2002.

${ }^{4} \mathrm{Al}$ presidente Clinton el Congreso le negó tres veces el Fast Track. De hecho, en toda la historia estadounidense sólo seis veces se ha concedido esta autorización al Ejecutivo: para la Ronda Tokio del GATT (1979), los ALC con Israel (1985), Canadá (1988), NAFTA (1993), la Ronda Uruguay (1994) y recientemente (2002) para negociar con Chile, Singapur, Centroamérica, Marruecos, la nueva Ronda OMC y el ALCA. 
negociaciones se efectuó un mes después del anuncio, mientras que el verdadero inicio requería del equipo del presidente George W. Bush. Una vez asumido el nuevo gabinete, se negoció durante todo el 2001 sin TPA (Fast Track), el que finalmente se obtuvo por diferencia mínima de votos en agosto del $2002^{5}$ y precipitó el cierre de las negociaciones cuatro meses después.

\section{III. ¿POR QUÉ Negociar un ACUERdo DE Libre Comercio bilateral.... Y CON EsTAdos Unidos?}

La apertura chilena se inició en la segunda mitad del '70. Para las autoridades económicas de la época, cualquier lógica que pudiera tener la estrategia de industrialización basada en la sustitución de importaciones (ISI), si alguna vez la tuvo, ya había llegado a rendimientos decrecientes y estaba agotada. Por el contrario, consideraban que el mercado interno de una economía pequeña como la chilena no permitiría lograr la especialización ni alcanzar las economías de escala para el desempeño eficiente de gran parte del aparato productivo. El ejemplo más dramático de esto fueron las 20 armadurías de vehículos que existían para un mercado total de apenas 30.000 automóviles al año y este tipo de aberración era más bien la regla más que la excepción. De hecho, el arancel medio chileno en 1973 bordea- ba el $100 \%$ y con alta diferenciación por rubros, sin más lógica que ofrecer a cada sector el nivel de protección necesario para mantenerlo rentable. De ahí que concluyeran que la protección y sus distorsiones eran la causa del escaso crecimiento de las exportaciones (nulo en términos per cápita) y del producto global (menos de $4 \%$ entre 1940-1973) y que liberalizar la economía era un imperativo.

\section{El aislamiento luego del golpe militar no permitía estrategias multilaterales.}

Por cierto, el aislamiento internacional tras el golpe militar y el estado de autarquía general en la región, aun inmersa en el proceso ISI, no permitía una estrategia bi o multilateral (incluso Chile se retiró del Pacto Andino en 1976), y dados los niveles y la estructura arancelaria chilenos, la apertura unilateral aparecía como la opción más aconsejable. Entonces el país emparejó y redujo los aranceles, que a mediados del 1979, ya estaban en $10 \%$ y aunque elevó las tarifas durante la crisis del ' 82 , al llegar el gobierno democrático se encontraban en $15 \%$ parejos.

Hubo actividades golpeadas por la apertura, pero cambiar la demanda local por la demanda internacional, le permitió al país especializarse en bienes competitivos internacionalmente y las exportaciones crecieron al $10 \%$ anual, de tal modo

${ }^{5}$ Algunas voces alertaron sobre asociarse a una potencia que entregaba un paquete adicional de subsidios agrícolas de 180 mil millones de dólares y sobre las restricciones que el TPA imponía al Ejecutivo, dejándole muy limitada capacidad de apertura al sector agrícola, incluyendo varias exportaciones clave de la región. Baste recordar los apenas tres votos en el Senado y uno en la Cámara que dieron la aprobación al TPA. 
que el volumen exportado de 1990 sería ¡cinco veces lo que era en 1973! Pese a críticas iniciales, el gobierno democrático de la Concertación adoptó y consolidó la apertura y la estrategia de desarrollo "hacia afuera", profundizándola en 1991 con la rebaja unilateral de los aranceles de $15 \%$ a $11 \%$ y otra vez en $1998^{6}$ cuando el Congreso aprobó un programa de desgravación (1 punto porcentual anual) que llevaría a un $6 \%$ de arancel general a 2003. Simultáneamente el país se embarcó en varias negociaciones comerciales y asumió los compromisos multilaterales de la Ronda Uruguay (Cuadro $\mathrm{N}^{\circ} 1$ ).

\section{Los bloques regionales son una alternativa a la multilateralidad.}

Antes de responder al caso puntual -¿por qué con Estados Unidos?-, veamos la pregunta ampliada. Los países negocian sabiendo que los acuerdos bilaterales son un sustituto imperfecto del libre comercio mundial, lo hacen porque es la mejor oportunidad viable y saben que un "segundo mejor" es suficiente cuando el óptimo no es alcanzable. Respuestas complementarias existen en lo político y lo estratégico, mientras los países puedan escoger los socios bilaterales más importantes.

La mejor alternativa -el libre comercio mundial- no está disponible, y a juz- gar por su lenta evolución será la próxima generación quien disfrute sus ganancias. La OMC, que administra el sistema multilateral, es una institución enorme y heterogénea, incapaz de acelerar el proceso y disipar la incertidumbre sobre su resultado final. La Ronda Uruguay demoró cinco años más de lo previsto (debía concluir en 1990 y lo hizo en 1995) y mantuvo importantes niveles de protección en los países desarrollados, se recuerda el fracasado intento de Seattle 99, y la actual Ronda de Doha presenta limitantes similares ${ }^{7}$. Sin ignorar su aporte al establecer reglas comunes, se puede anticipar un limitado avance y un largo horizonte de consolidación, lo que sugiere la necesidad de una agenda bilateral activa que beneficie al país mientras el proceso mundial se consolida a su ritmo.

En cuanto a los bloques regionales -una alternativa a la multilateralidad- un paralelo de la integración entre América y Europa puede mostrar estas velocidades diversas, quizás justificables por madurez e historia, pero no por la teoría del comercio. En 1960 surgió la Asociación Europea de Libre Comercio y también su homóloga Latinoamericana; treinta años después se creaban el Área Económica Europea $^{8}$ y el Mercosur, en 1993 se firmó el Tratado de Maastricht y a principios del 1994 el Nafta. La Unión Europea tiene moneda y Banco Central únicos, ciudadanía compartida y carece de fronteras,

\footnotetext{
${ }^{6}$ Con el objetivo explicito de evitar la desviación de comercio generada como resultado de la incorporación al Mercosur.

${ }^{7}$ Para un completo análisis de la OMC y la participación chilena, ver Sáez 1999.

${ }^{8}$ Todos los países europeos, incluso los no comunitarios, están dentro de un área económica única, con reglas y obligaciones parejas, con apertura de fronteras, etc.
} 
algo que el incierto ALCA jamás alcanzará. Esto Chile lo sabe bien, pues en dos años logró acuerdos de libre comercio con toda Europa (Cuadro $\mathrm{N}^{\circ} 1$ ), mientras que treinta años de integración latina han resultado frustrantes.

El libre comercio mundial no es un fin, sino un medio para aumentar la eficiencia y el bienestar global y los acuerdos de libre comercio son simplemente otro medio que persigue el mismo fin. En definitiva, los acuerdos con socios seleccionados por su participación en el comercio bilateral -global o sectorial- tienen ventajas porque permiten compromisos más profundos, son más fáciles y rápidos de negociar, reducen los costos de esperar a la OMC y anticipan el clima de liberalización.

Frankel y Romer (1999) analizan los efectos del comercio sobre el crecimiento. Según ellos, existen varias vías que relacionan positivamente comercio y crecimiento, e incluso estas se acentúan cuando un país pequeño se asocia a uno grande. La mayoría de los beneficios estáticos (por una vez) pueden obtenerse de la apertura unilateral que ya Chile practicó: menos proteccionismo permite especializarse y esto genera más eficiencia en la asignación y el empleo de los recursos. Pero un acuerdo de libre comercio es mucho más que esto, pues reduce los aranceles así como otras barreras que limitan el comercio y que una vez eliminadas permiten una relación más cierta y con menores costos de transacción. También generan efectos dinámicos (permanentes) en la productividad y un proceso endógeno con aumentos de capital, externalidades positivas, efecto de desbordamiento de tecnología y capital humano (por el intercambio y la inversión), lo que permite mayores tasas de crecimiento, con altos niveles de desarrollo y bienestar.

\section{Chile obtendrá sus primeras ganancias de la rebaja arancelaria.}

Para Chile, las primeras ganancias llegarán por la rebaja arancelaria, que permite mayor variedad y menores precios, tanto de bienes finales que amplían las oportunidades de consumo, como de bienes intermedios que incrementan las capacidades productivas internas. En nuestro Tratado, el $88.5 \%$ de volumen $(90 \%$ de los bienes) importado por Chile desde Estados Unidos recibirá desgravación inmediata, es decir el mismo día de su entrada en vigor dejará de pagar el $6 \%$ de arancel general, el calendario se consolida cada bienio y doce años después del inicio se liberaliza el saldo ( $2.4 \%$ en valor, $1,2 \%$ de productos) y se establece plenamente el área de libre comercio.

Ante el shock competitivo, los productores internos deberán elevar su productividad, ya sea por medios tecnológicos o por capital humano, pero definitivamente se mantendrán en el umbral de la eficiencia. Esto, que económicamente es muy deseable y una ganancia importante, será considerado un daño y no un beneficio por los opositores al libre comercio, porque estos bienes más baratos, de mayor variedad y calidad, son importaciones que "desplazan" productos nacionales. En una visión mercantilista, las 
importaciones son un mal porque obligan a los productores locales a competir, lo que, aseguran, sólo es posible bajando los salarios y las condiciones laborales, precarizando el empleo y aumentando el desempleo. La visión positiva prevé que las oportunidades de negocios creadas por la apertura y la especialización basada en la eficiencia, permitirán absorber los recursos liberados por los sectores menos competitivos. De todas maneras, puede descartarse el temor de ser "inundados" por productos importados. Sin considerar que la estructura del intercambio comercial con Estados Unidos nos hace importar bienes de capital y tecnologías avanzadas que Chile no produce, en definitiva lo que determina el aumento de las importaciones en Chile es nuestra propia capacidad de exportar y son las variaciones del tipo de cambio lo que garantizan ese resultado. Finalmente no existe "destrucción" ni pérdida neta de capacidad productiva, sino que un cambio en el patrón de producción y comercio producto de la mayor eficiencia interna y las demandas que se enfrentan en el exterior.

De ahí que si bien el libre comercio y sus aproximaciones bilaterales generan perdedores y ganadores, beneficiados y desplazados, las ganancias compensarán las pérdidas con la reubicación de recursos al sector exportador. Esa es la esencia del libre comercio: elevar la producción en los sectores donde cada país produce con mayor eficiencia y reducirla en los demás, habrá costos por un tiempo, pero al final la economía y el bienestar crecerán.

Lo más complejo del fenómeno son sus efectos asimétricos durante la transición. En la práctica, los beneficios se dispersan mientras que los costos se concentran, muchos ganan poco -millones que compran más barato bienes, insumos y capital-o no tan poco -miles de empresas exportadoras- pero pocos pierden mucho -cientos de productores y sus miles de empleados que compiten con importaciones-. Aunque en definitiva las ganancias superan las perdidas, algunas empresas podrían no soportar la competencia, deberán cerrar y cambiar de rubro?.

\section{Algunas empresas deberán cerrar y cambiar de rubro por la presión de la competencia.}

Este proceso de dislocación y reajuste es complejo y muchas veces lento. En ocasiones, la concentración geográfica (v.g. agrícola) dificulta la posibilidad de reasignación y genera efectos colaterales (v.g. migración a las urbes). Adicionalmente, los "perdedores" tienen menores costos de organizarse -son menos- y pronto son vistos protestando contra la globalización, asociados a grupos de poder con influencia política y alcanzar desproporcionada representación en el Congreso. Esta realidad se manifiesta permanentemente en las negociaciones co-

\footnotetext{
${ }^{9}$ Esto es poco probable en Chile, donde los aranceles bajan de $6 \%$ a $0 \%$, siendo un margen manejable de ajuste. Quienes se verían más afectados serían aquellos que emplean otros mecanismos para sobrevivir, como las bandas de precios, que establecen costos mínimos de importación al azúcar, trigo, harina y aceites comestibles.
} 
merciales y se materializa con calendarios de desgravación "especiales", excepciones o cláusulas ad hoc para ciertos sectores. Como veremos, nuestro TLC dispone de doce años para la liberalización completa del sector agrícola, mientras que el sector industrial lo consigue en 2 años. ¡Diez años más! requieren los sectores "sensibles" para adaptarse a la competencia. Al menos en este caso es una fracción mínima del comercio $(4,5 \%)$, comparada con la protección que mantuvo el ALC con Corea del Sur, en virtud de lo cual más de la mitad del comercio se desgrava a diez años.

Los países grandes tienen demandas y mercados internos muy desarrollados y competitivos, por eso resulta más beneficioso para un país pequeño asociarse a uno grande -el caso en cuestión-. Un gran mercado internacional permite mantener un tamaño de planta que sería imposible con la demanda interna chilena, lo que posibilita aprovechar economías de escala. En economías muy distorsionadas, un acuerdo comercial con un socio de esta magnitud podría cambiar los patrones de comercio o la "localización" productiva ${ }^{10}$, pero un país abierto con mínimas distorsiones como lo es Chile -las bandas de precios son la única excepciónno debe temer estos efectos y en cambio puede esperar la profundización del nivel actual de participación de mercado, la apertura de nuevos nichos diversificando las exportaciones y el incremento del valor agregado exportado.

\section{Los efectos del tratado serán positivos para las inversiones y para la reasignación de recursos.}

Puede preverse que los efectos en las inversiones también pueden anticiparse positivos, porque el ALC "certifica" las políticas chilenas y las proyecta a futuro, lo que consolida la diferenciación del país y mantiene reducido el riesgo asociado a invertir en Chile. Esto no sólo atrae inversión extranjera, también permite a los empresarios chilenos obtener créditos a menor costo y profundizar el mercado de capitales doméstico.

El resultado final será como el de todo proceso aperturista: transferencia y reasignación de recursos entre países y dentro de ellos, para un juego de "suma positiva" donde ambos ganan en eficiencia y bienestar. Si la bilateralidad se da entre un país grande y otro pequeño, este último tiene ventajas adicionales que aprovechar del otro mercado y ganará aún más si lo hace.

\section{.....y por qué una asociación \\ Chile-Estados Unidos}

Detalles aparte, este Tratado coloca a Chile en la exclusiva lista de países que han suscrito acuerdos de libre comercio con Estados Unidos: Israel (1985), Canadá (1989), México-Canadá (1994) y Jordania $(2001)^{11}$. Un listado que combi-

\footnotetext{
${ }^{10}$ Como las maquilas en la frontera Estados Unidos-México tras el Nafta.

${ }^{11}$ Los TLC de Singapur y Chile esperan ratificación parlamentaria; Centroamérica y Marruecos han comenzado ya su negociación.
} 
na evidentes razones geopolíticas, con otras comerciales. Canadá ha sido su principal socio comercial y el origen de casi $20 \%$ de las importaciones norteamericanas (que más que se duplicaron entre 1993-2000), mientras México ocupa el segundo lugar desde que desplazó a Japón en 1999. Chile, en cambio, no tiene atractivo comercial, es un $0,3 \%$ de las importaciones y un $0,5 \%$ de las exportaciones estadounidenses, pero es un socio importante en la ampliación comercial latinoamericana. Si la política de Estados Unidos hacia la región es la que esbozó en la primera Cumbre de las Américas y pretende hacerlo a través del ALCA, entonces este Tratado y sus repercusiones son un paso clave en su consecución.(Ver Cuadro 2, en Anexos.)

\section{La lógica chilena}

Estados Unidos es la primera economía mundial. Un mercado de 282 millones de habitantes con ingresos per capita sobre los 35.000 dólares, que representa más del 20\% del PIB global y es el país industrializado de mayor crecimiento. También ha sido el principal socio externo de Chile por más de veinte años, con un nivel de intercambio comercial que en la década pasada superó los 5.000 millones de dólares en promedio, recibió el $17 \%$ del total exportado por Chile y originó el 21.2\% importado. En inversión extranjera directa, entre 1974-2000 se han materializado en nuestro país 13.545 mi- llones de dólares provenientes de Estados Unidos, que lo convierten en el principal inversionista externo, con un 31\% del total. En el contexto americano, Estados Unidos es el 60\% de la economía del continente, mientras Chile es apenas un 4\%. (Ver Cuadro 3, en Anexos.)

\section{La firma de acuerdos genera tanto efectos deseables como adversos.}

Como veremos, el Tratado otorga un trato privilegiado a Chile que mejora las posibilidades de acceso a los exportadores, principalmente por la rebaja arancelaria a los productos con mayor valor agregado. Aunque los principales productos exportados (más del $80 \%$ del total) al año 2000 ya ingresan a Estados Unidos con arancel ad valorem cero y de ellos un $75 \%$ sin aranceles específicos ${ }^{12}$, el Tratado garantiza la eliminación de todos los aranceles vigentes y principalmente suprime otras barreras muy importantes que no son arancelarias.

La estrategia chilena de acuerdos comerciales, que lo llevan a negociar con toda Europa, la mayor parte de América y socios selectivos de Asia, genera algunos efectos deseables y otros adversos. Las preferencias bilaterales tienen consecuencias para el comercio y las inversiones, que pueden ser al mismo tiempo positivos, por creación o negativos, por desviación. Para eludir los costos se debe diversificar el número de socios y priorizar de acuerdo con la participación en el in-

${ }^{12}$ Banco Central, pág. 215. 
tercambio comercial y de inversiones; por eso se espera que los costos por "desviación" sean mínimos con Estados Unidos y que adicionalmente profundice los beneficios de los Acuerdos con Europa.

\section{Una estrategia de apertura bilateral amplia eleva el bienestar del país.}

Para asegurar que los efectos positivos superen los negativos, además de la ausencia de barreras externas a otros socios, que Chile no emplea por tener arancel general parejo, influyen las características del socio bilateral. El efecto "creación de comercio" será mayor siempre que el socio tenga un mercado grande y dinámico, sea un productor eficiente, proveedor de inversión extranjera directa y tecnología moderna, macroeconomía y moneda estables, altas barreras al comercio y esté geográficamente próximo. Con estos criterios, Estados Unidos es un socio que permite prever mínima desviación y efecto neto positivo; no es sólo el principal socio comercial e inversionista en Chile, también es la economía industrializada más dinámica y estable del mundo y está más cerca que Europa o Japón, aunque estos últimos resultan atractivos por tener barreras muy importantes.

Larraín y Coeymans (1994) estimaron que el PIB chileno sería $10 \%$ mayor en el largo plazo (15 años) gracias al TLC con Estados Unidos. Según los autores, el efecto más importante es que los compromisos del tratado actúan como un "seguro de estabilidad" de la política económica y eso reduce la prima por riesgo y la tasa de in- terés que enfrentan los inversionistas, aumentando la inversión extranjera e interna en Chile. Este "seguro" acota las opciones de política a lo que el Tratado permita y aunque en la práctica se consolida el nivel actual de compromisos sin que haya necesariamente una mejora, dicho congelamiento impide que el gobierno modifique hacia el futuro sus políticas con leyes o regulaciones más restrictivas. Un impacto menor sobre el crecimiento se genera por la rebaja arancelaria.

Buscando ganancias sobre el nivel de bienestar chileno, Harrison, Rutherford y Tarr (1997) evalúan un conjunto de opciones "aditivas" en la política comercial chilena. Este análisis permite comprender mejor los impactos de la liberalización bilateral, haciendo notar el efecto neto entre creación y desvío de comercio. El Cuadro $\mathrm{N}^{\circ} 4$, en Anexos, cuantifica estos efectos. Asociarse únicamente al Mercosur (segunda columna) reduce el bienestar porque la desviación supera a la creación de comercio, mientras que incorporarse al NAFTA tendría efecto positivo pero muy menor. Las columnas hacia la derecha agregan nuevos socios bilaterales y cada vez a un costo menor en desviación y mayores ganancias en bienestar. El impacto del TLC con Estados Unidos se aprecia en las dos últimas columnas.

Del estudio pueden extraerse dos importantes conclusiones. Primero, que una estrategia de apertura bilateral amplia eleva el bienestar del país. Segundo, negociar un acuerdo comercial que no liberalice el universo total de bienes y en cambio excluya sectores y productos, reduce fuertemente los beneficios de la negociación, 
puede que el país aumente sus exportaciones y aun así el bienestar se reduce. Ambas validan la política comercial chilena de negociar acuerdos de libre comercio sin excluir sectores y con varios socios.

\section{La transición pacífica hacia la democracia fue uno de los atractivos chilenos.}

\section{La lógica norteamericana}

Ya mencionamos los cuatro acuerdos de libre comercio suscritos por Estados Unidos, selección en que predomina un interés político del que Chile no escapa. A nivel agregado, el intercambio comercial con Chile es insignificante para Estados Unidos y es fácil prever que su efecto en el PIB de ese país será imperceptible ${ }^{13}$. De hecho, Chile es a Estados Unidos lo que Dinamarca o Filipinas para Chile, apenas un 0.4 del comercio y el socio número 38 .

La transición pacífica a la democracia puede haber sido uno de los atractivos chilenos. El Tratado sería visto por el mundo como un premio al proceso y un respaldo al nuevo gobierno. Más aún, dados la madurez política, el manejo macroeconómico, la apertura comercial y de inversiones, las privatizaciones y el crecimiento impresionante de la primera mitad de los '90, Chile era de los pocos países donde las recetas del Consenso de Washington parecían efectivas y ejemplarizantes: ¡si hasta tenía superávit fiscales! La teoría del "premio" otra vez.

Mientras termina el siglo XX las medidas "neoliberales" han caído en entredicho y desuso. Muchos consideran su fracaso una muestra más del inviable camino capitalista, al menos la desilusión popular se contenta con esta explicación y emergiendo de la inestabilidad políticoeconómica aparecen los neopopulis-mos siempre antiestadounidenses y antimercado. Como es tradición regional, las culpas recaen en Estados Unidos, los teóricos de la conspiración lo acusan de hegemonía, extrema penetración, intervención y saqueo; otros le atribuyen nulo liderazgo mundial en la materia, pues de casi 130 acuerdos firmados en la década, Estados Unidos tiene apenas 4 (México en cambio ha firmado 22) y de su lento paso por el ALCA como factor de retraso en la apertura regional, que demora su liberalización para ofrecer "más" y lograr mejor acceso a Estados Unidos.

Las autoridades estadounidenses saben cuán apetecido es su mercado, e históricamente han dosificado su apertura con estrategias políticas dirigidas a una extensa lista de países que quisieran ser "invitados" a un proceso de asociación y liberalización mutuo. Así, ha empleado unilateralmente el Sistema Generalizado de Preferencias ${ }^{14}$ (SGP) para inducir y

\footnotetext{
${ }^{13}$ Aunque hay sectores en que los proveedores chilenos son muy importantes. El $16 \%$ importado de frutas es chileno (sólo superado por México con un 18,5\%), los vinos o pescados ocupan el $6 \%$ y maderas o frutas en conserva el $3 \%$.

${ }^{14}$ Un mecanismo de los países desarrollados, a través del cual benefician a los subdesarrollados con rebajas arancelarias unilaterales. Estas preferencias son temporales, renovables y pueden ser modifica-
} 
premiar ciertos "logros" en los países subdesarrollados. Esos logros del SGP incluían por ejemplo cláusulas laborales. Ahora en cambio planea utilizar estos acuerdos amplios para imponer ( $¿$ acelerar?) cambios institucionales profundos en los países en desarrollo y puesto que los acuerdos comerciales se transforman en ley dentro de cada país, los acuerdos le permiten al gobierno de Estados Unidos transformarse en colegislador de sus socios, "guiándolos" hacia la institucionalidad requerida en el libre mercado ${ }^{15}$.

\section{El libre comercio es un concepto general y no un sesgo partidista 0 ideológico.}

En esta cruzada pro-mercado es muy importante el efecto "precedente" porque, aunque la tendencia empieza a cambiar, normalmente los acuerdos bilaterales se dan en el ámbito "norte-norte" o "sur-sur", evidenciando la dificultad de negociaciones entre países de diverso estado de desarrollo. Si Estados Unidos quería demostrar la viabilidad de acuerdos "norte-sur", para revertir su ausencia e imponer cambios institucionales a través de tratados comerciales amplios, disciplinas más estrictas que la OMC y compromisos sociales (laborales y de medio ambiente), necesariamente debían conseguir un socio del "sur" capaz de enfrentar el desa- fío y salir airoso. De acuerdo con ese criterio Chile es un objetivo estratégico, el candidato natural para redactar un acuerdo "base" que corrija las deficiencias del NAFTA, indique a los que se resisten al ALCA (Brasil principalmente) que para Estados Unidos la bilateralidad es también una alternativa y le señale quienes quieran acceder a su mercado, que espera que den algo a cambio.

Asociarse primero a Chile, uno de los países más abiertos (como Singapur en Asia) es más fácil que lidiar con un socio proteccionista, y permite incorporar una serie de compromisos "base" que serían muy difíciles de aceptar para otros latinoamericanos (o asiáticos), pero que ahora Estados Unidos pondrá "sobre la mesa" como un nivel "mínimo" de cualquier futuro ALC. También se aplica en sentido contrario, lo que Estados Unidos no "entregó" a pesar de los compromisos asumidos por Chile (v.g. el levantamiento de los mecanismos antidumping), no se lo entregará a otros.

Por último, y aunque sea más accidental que provocado, llama la atención que la brecha ideológica entre los presidentes Lagos y Bush sea la más amplia de los pasados gobiernos respectivos: un "socialista" y un "republicano". Esto en definitiva demuestra que el libre comercio como opción de política económica es ya un concepto general y no un sesgo partidista o ideológico.

das sin aviso ni explicación, de modo que siempre está presente la incertidumbre de invertir a partir de un compromiso frágil. Chile recibía estas preferencias temporales, que tras el TLC han quedado consolidadas y llevadas a cero en forma permanente.

${ }^{15}$ Para un análisis de estos sobre la institucionalidad mexicana, ver López-Córdova. Para la teoría respectiva, ver Rodrik 2000. 


\section{Qué dice el Tratado de Libre Comercio entre Chile y Estados UNIDOS}

Enfrentarse al texto del TLC no es tarea fácil; como toda ley que finalmente será, está construida y dominada por conceptos legales que especifican los detalles (muchos y hasta un nivel mínimo) con acuerdo a las cuales se regirán estos nuevos socios. La composición del equipo negociador (más de 50-60 por país) estaba fuertemente marcada por abogados y economistas, provenientes de diversas dependencias públicas. Salvo un par de capítulos, la vocería en las negociaciones recayó en la Dirección de Relaciones Económicas (DIRECON) de la Cancillería chilena y su homóloga Oficina del Representante de Comercio (USTR en la sigla inglesa), de la Oficina Ejecutiva del presidente de Estados Unidos, quienes a su vez tenían un equipo multidisciplinario y multiagencial de apoyo. (Ver Cuadro 5, en Anexos.)

\section{El Tratado afecta transversalmente todas las materias.}

El Tratado consta de 24 capítulos, algunos de ámbito genérico o que afectan todas las materias transversalmente, mientras que los restantes abarcan asuntos específicos. En total suman 19 temas de negociación agrupadas en siete áreas: Comercio de Bienes, Mecanismos de Defensa Comercial, Normas y Estándares, Servicios e Inversiones, Asuntos Vinculados al Comercio, Institucionales, Laborales y
Medioambientales. Nuestro análisis intentará proveer al lector de una visión inicial del texto, enfatizando los asuntos más importantes e pasando por alto los detalles.

\section{Comercio}

Analicemos los tres primeros capítulos específicos, aquellos que regulan el tratamiento que recibirán los productos originarios que las partes intercambien: Trato Nacional y Acceso a Mercado de Bienes, Reglas de Origen y Administración Aduanera. En el primero se definen las rebajas arancelarias, que es la esencia de un Acuerdo comercial y su cara más visible, donde se determina el calendario de desgravación que llevará al libre comercio entre las partes. Para beneficiarse de estas rebajas, el producto debe cumplir ciertas "reglas de origen", que aquí se negocian para evitar que terceros países se beneficien del Acuerdo (se exige componente nacional y aporte local en el valor, grado de procesamiento, etc.); reglas estrictas reducen el universo de bienes que se beneficiarán, afectando principalmente aquellos con insumos importados y bajo nivel de procesamiento. Por último, se define el nivel de trato, eficiencia, transparencia y agilidad que se espera caracterice el desempeño de las Aduanas respectivas, a partir de procedimientos simplificados y uso de tecnología, resoluciones anticipadas y criterios mutuos de valoración, que reducirán los costos de transacción y espera en las fronteras.

En materia de "Acceso", lo más destacable es la desaparición total de aranceles, cuotas y otras restricciones en el 
intercambio mutuo en doce años, para los bienes que cumplan con la norma de "origen". Los doce años son necesarios para el sector agrícola, pero en dos años se libera todo el comercio no agrícola. Como resume el Cuadro $\mathrm{N}^{\circ} 6$ (en Anexos), a nivel global se desgravará inmediatamente el $87 \%$ del intercambio (equivale a un 95\% de los bienes), a los dos años habrá un $95 \%$ del comercio sin aranceles y cuando termine el calendario de desgravación (12 años) todo el universo de bienes originarios ingresará a ambos países con arancel cero ${ }^{16}$.

Como hemos dicho, la mayoría de los productos chilenos ya ingresan a Estados Unidos con arancel cero, bien porque dicha tarifa se aplica a todo el mundo o porque el SGP lo libera del pago. Por eso, el impacto mayor en términos arancelarios está en productos que actualmente Chile no exporta, aquellos con mayor valor agregado y procesamiento. Es que la configuración arancelaria de los Estados Unidos sigue un "escalonamiento", vale decir, el arancel se eleva a medida que aumenta el nivel de procesamiento y se agrega valor al producto. Con la eliminación de todos los aranceles, Chile podrá aumentar el grado de procesamiento de los bienes que exporta, sin tener que pagar mayores aranceles por ello.

Adicionalmente, incluso cuando la tarifa estadounidense sea cero para todo el mundo, el TLC entrega ventajas a los exportadores chilenos, haciéndolos más atractivos en una relación comercial respecto de otros países. Por el tratamiento expedito en Aduana, los compromisos en normativa técnica, el mecanismo propio de resolución de conflictos y en general por el resto de los compromisos bilaterales, operar con Chile tendrá menores costos de transacción y mayor certidumbre que operar con otros competidores chilenos.

\section{El impacto arancelario estará en productos que ahora Chile no exporta a Estados Unidos.}

Como se anticipaba, las sensibilidades más importantes se presentaban en los productos del agro, y allí se manifestaron las presiones de las partes interesadas que lograron mantener protección (en ambos países) y diferir parte de la liberalización agrícola a un período de 12 años, aunque el grueso (84\%) alcanza arancel cero inmediatamente. Chile mantiene por 12 años su sistema de bandas y el uso del drawback, aunque ambos se van desmantelando y desaparecen finalmente el año 12. Por su parte, el impuesto al lujo de los automóviles se reduce hasta desaparecer al cuarto año. En Estados Unidos varios productos quedarán bajo un sistema de cuotas que restringen las exportaciones chilenas, que se ajustan (crecen) cada año y se eliminan a lo largo del calendario ${ }^{17}$. Efectivamente los

${ }^{16}$ La única excepción respecto de Estados Unidos es el tabaco. El azúcar tiene un mecanismo especial de desgravación en ambos países.

${ }^{17}$ Los lácteos, por ejemplo, reciben una cuota libre de arancel de 3.500 toneladas, que crece $7 \%$ cada año y desaparece a los 12 años; las paltas tienen 49.000 toneladas de cuota, que crece $5 \%$ anualmente y se libera del todo al año 12, otras (carnes) se liberan de cuotas al cuarto año, etc. 
plazos pudieron ser menores y los porcentajes mayores, pero para ser objetivos, es un magnífico nivel de acceso para el sector exportador, que salvo un mecanismo especial para el azúcar y el tabaco, eliminará todas las tarifas, cuotas y restricciones actuales.

\section{Novísimos}

Que el TLC con Estados Unidos marca una nueva generación se demuestra en los compromisos sobre comercio electrónico, el primer capítulo mundial para mantener el ciberespacio libre de aranceles y barreras. Las Partes se comprometen a no aplicar aranceles a los productos digitalizados que sean trasmitidos por medios electrónicos o vengan sobre un medio físico portador, y a no discriminar entre estos bienes y otros del mundo físico. Otra parte importante del acuerdo se relaciona con la cooperación mutua.

El Capítulo de Contratación Pública es un logro extraordinario de la negociación y acaso el único que puede asegurarse como creación pura de comercio respecto de Chile. La situación es simple: desde la década del '30 el Buy American Act prohíbe al Gobierno Federal de los Estados Unidos comprar bienes provenientes del extranjero, de modo que los productos chilenos eran "no elegibles" para ser adquiridos con el presupuesto estadounidense. La única forma de romper dicho impedimento y acceder con certeza a este mercado enorme y cerrado, es por medio de este capítulo y así se hizo, ampliándolo más allá del Gobierno Federal a varios Estados $(34)^{18}$. Aunque aún se aplican restricciones sobre algunos bienes, preferencias locales (v.g. veteranos, pymes, etc.) y los beneficios se perciben sobre un umbral (50 mil dólares), cualquier proveedor chileno puede ser elegido para vender a las instituciones federales o estaduales, recibiendo el mismo trato que un ciudadano estadounidense.

\section{El tratado también abarca diversos aspectos del comercio electrónico.}

\section{Aspectos controvertidos}

Ninguna parte del TLC ha sido tan discutida como la de Asuntos Laborales y Ambientales, tanto en Chile como en Estados Unidos. Desde los primeros contactos Chile advirtió que no los quería "dentro" del Tratado y que prefería la solución de "Acuerdos Paralelos", que obtuvo con Canadá; tampoco aceptaría sanciones comerciales y era necesario un enfoque de "cooperación"19. Esta es la

${ }^{18}$ El gasto total del gobierno en 1998 fue de US\$ 1.49 trillones, un $32 \%$ del PIB. El gasto a nivel federal, sólo en bienes, fue de US\$ 35,5 billones y a nivel estatal US\$ 94.5 billones.

${ }^{19}$ Esto, junto a la eliminación del antidumping, eran los objetivos más explícitos y más importantes para las autoridades chilenas, no se sabe si por estrategia de "anuncios" o por convicción. El antidumping es uno de los mecanismos de defensa comercial que más emplea Estados Unidos y que podrá seguir aplicando a Chile pues no se logró limitar en el TLC, como si se hizo en el Acuerdo de Libre Comercio Canadá-Chile. 
fórmula Nafta de abordar la temática, que el propio Estados Unidos impulsó en su oportunidad, pero que ahora deseaba modificar por las críticas de los sectores sociales, sindicales y ambientalistas a los mecanismos y las exigencias del acuerdo con México y Canadá. Los empresarios locales anticipaban un desastre con su inclusión y hasta la Cámara chileno-norteamericana de Comercio se oponía. El resultado final da cuenta de la importancia política de este tema en Estados Unidos, que no se empezó a negociar efectivamente hasta que el TPA (que lo incluye como requisito sine qua non) estuvo aprobado y que finalmente terminó dentro del Tratado, con multas de hasta 15 millones de dólares y sanciones comerciales (eliminación de las preferencias del TLC) como medidas de último recurso.

\section{Deben respetarse las leyes particulares en cuanto a estándares de calidad y aspectos laborales.}

El debate respecto de la relación entre ambos temas y los asuntos comerciales es antiguo, aunque su primer antecedente explicito está en el Nafta ${ }^{20}$. Existe en él una conjunción de intereses sociales, proteccionistas y sindicales para elevar los estándares laborales, que hacen muy difícil obtener un equilibrio razonable. Los denominados grupos de la "sociedad civil" están preocupados del tra- bajo infantil, el trabajo forzoso y las condiciones laborales mínimas (salario, duración de la jornada y seguridad); en esto último coinciden los sindicatos, que además quieren elevar artificialmente su poder negociador y atraer miembros, porque les interesa el derecho de asociación (sindicalización) y la negociación colectiva. Quienes temen la apertura apoyan estos y otros reclamos que aumenten los requisitos y disminuyan la competitividad chilena.

Las Partes aceptan que cada una tiene derecho a fijar los estándares, aunque ellos deben ser consecuentes con los previstos por las organizaciones internacionales y deben elevarse según evolucionen los tiempos, pero no se impone a Chile los estándares aplicables a Estados Unidos. Esto significa que el compromiso de las partes es cumplir su propia legislación en la materia, pero no aumentarla, aunque se comprometen a no relajarla para atraer inversiones o crear comercio ${ }^{21}$. La diferencia es sutil pero igualmente impone un compromiso: el gobierno no puede dejar de hacer cumplir la ley en cinco materias específicas relacionadas con los estándares laborales que el TPA exige, esto es el derecho de asociación, de negociar colectivamente, la prihibición del trabajo forzoso, la edad mínima en el empleo y la eliminación de las peores formas del trabajo infantil; la aplicación de condiciones de trabajo aceptables respecto del salario mínimo, jornada y seguridad en el trabajo.

${ }^{20}$ El clamor por su inclusión (junto a medio ambiente) en las rondas de la OMC y la negativa de dicha institución, provocó el fracaso de Seattle en 1999.

${ }^{21}$ En esta y en otras materias existe una asimétrica relación de compromisos, pues Chile asume el cumplimiento de toda su legislación, mientras que Estados Unidos no compromete la legislación estadual. 
El caso medioambiental es igual en los compromisos, la aplicación y los montos, aunque no existen estos estándares mínimos. En resumen, cualquier "acción o inacción" respecto de las leyes ambientales internas puede impugnarse si causa daño al comercio de las Partes. Tampoco pueden relajarse las actuales exigencias para promover el comercio o atraer inversiones. El Capítulo Ambiental incluye un anexo con programas específicos de cooperación entre ambos países.

\section{El Tratado generará un ajuste en el poder sindical.}

En concreto existen dos compromisos. Primero, la limitación respecto de la reducción de las exigencias laborales y ambientales con el objeto de crear condiciones más competitivas o dejar de fiscalizar el cumplimiento de la normativa actual, para exportar a Estados Unidos. Segundo, el compromiso adicional en términos laborales de hacer cumplir los derechos fundamentales de los trabajadores, resumidos en esos cinco principios. Cualquier violación sólo puede ser reclamada por Estados Unidos si como resultado de un "curso de acción o inacción sostenido y recurrente" de incumplimiento, se afecte el comercio entre las partes. Ambos capítulos tienen un mecanismo propio de consulta, con un Panel de expertos y mecanismos de cooperación antes de llegar al pago de multa, que debe ser proporcional al daño pero no mayor superior a $15 \mathrm{mi}-$ llones de dólares y que una vez pagada (de no pagarse se eliminan los beneficios del TLC), se reinvierte en el país sancionado para corregir la falla.

Un impacto complejo en materia laboral es el ajuste que provocará respecto del poder sindical. Tal como asistimos al quiebre de otras instituciones ${ }^{22}$ por la oposición de objetivos entre el sector exportador y el que compite con importaciones, asistiremos a un quiebre del equilibrio dentro del mercado laboral y sindical entre las empresas que tienen relaciones comerciales con Estados Unidos y las que no las mantienen, pues sólo las primeras podrán apelar al Tratado en sus negociaciones. En definitiva, corremos el riesgo de que estas herramientas se instrumentalicen y sean usadas para hacer política sindical doméstica, ante la amenaza (al país, no a la empresa) de una multa millonaria, en lugar resolverlos en la Dirección del Trabajo u otras entidades del organigrama gubernamental chileno.

En materia ambiental, un tema delicado es la relación entre nuestra legislación y la canasta exportadora, intensiva en recursos naturales. Alrededor de un 90\% de nuestras exportaciones a Estados Unidos tiene vinculación directa con el medio ambiente: un tercio agrícola, otro tercio minero y el último se ordena en importancia por pesca, forestal y finalmente un $10 \%$ de bienes industriales. Las inversiones y la consolidación de estos sectores estará siempre muy vinculada con la normativa ambiental y con las exigencias que ella imponga a cada proyecto.

\footnotetext{
${ }^{22}$ La Sociedad Nacional de Agricultura.
} 
En conclusión, el principal impacto de los compromisos laborales y ambientales recaerá sobre el Congreso chileno, que deberá reflexionar con otro criterio e incluir este factor en cualquier modificación que pretenda agregar a nuestra normativa en ambas materias, partiendo de la base de que todas ellas serán recurribles por los Estados Unidosy deberán cumplirse cabalmente. Los entes fiscalizadores, como la Dirección del Trabajo y la Comisión Nacional del Medio Ambiente, también juegan un rol determinante susceptible de transformarlos, pues a ellos corresponde la tarea de hacer cumplir la ley.

\section{Los compromisos asumidos en Inversiones aseguran un nivel de protección dentro de un marco seguro y predecible.}

Servicios

El sector se servicios es parte creciente del producto en cada país y de su intercambio en comercio internacional. Se regula en varios capítulos, entre ellos, naturalmente, Comercio Transfronterizo de Servicios, Servicios Financieros, Telecomunicaciones, Entrada Temporal, Inversiones y también Comercio Electrónico. En varios sectores se otorga trato nacional y no discriminatorio a los proveedores de la otra Parte e incluso el trato de nación más favorecida, es decir, si uno de los países negocia con otro socio y le entrega un trato mejor, el otro recibe automáticamente dicho beneficio.

Se asume un compromiso amplio de abrir los mercados de servicios, los que podrán ser provistos trasfronterizamente (v.g. Comercio Electrónico), con movimientos del proveedor o el consumidor (Entrada Temporal) o porque se instala en el territorio de la otra parte para suministrarlo (Inversión). Existen compromisos de "igualdad regulatoria" independiente del origen del proveedor, es decir el regulador no puede discriminar por nacionalidad y debe mantener las mismas exigencias. Incluso se asumieron compromisos de transparencia en virtud de los cuales las Partes deben ser consultadas y escuchadas antes de los cambios en materia regulatoria.

Los compromisos asumidos en Inversiones aseguran un nivel de protección dentro de un marco seguro y predecible, con los mismos derechos y exigencias que las inversiones de origen nacional. Adicionalmente, definen con claridad al inversionista y sus derechos, evitando demandas frívolas de "ilusos" y demandas contra el Estado como las que desestabilizaron el Nafta por expropiación indirecta. Se espera que los compromisos asumidos por Chile, que en realidad consolidan el nivel actual, atraigan mayores inversiones estadounidenses a nuestro país, otorgando mayor certidumbre y credibilidad a la institucionalidad de las inversiones hacia el futuro. 


\section{Flujos de capital}

En la última ronda negociadora se resolvió uno de los temas más complejos de la negociación, que mantenía al Ministerio de Hacienda y al Banco Central chileno buscando una solución que mantuviera las atribuciones del Central. El tema es clave para evitar la volatilidad que crean los flujos especulativos de corto plazo y como herramienta cambiaria y de estabilidad macroeconómica, el Central aplicó un "encaje" que encarecía el movimiento de capitales. Entre enero de 1996 y junio de 1998 el encaje llegó a 30\%, para reducirse a $10 \%$ y luego a $0 \%$ en octubre de ese año. Aunque en la actualidad no se aplica (el encaje es de $0 \%$ ) es una de las atribuciones que la ley otorga a la autoridad monetaria ${ }^{23}$.

La controversia se tornó de alto nivel político y académico. De Chile viajó el Ministro de Hacienda Nicolás Eyzaguirre, ex Gerente de Estudios del Banco Central; su contraparte norteamericana era John Taylor, subsecretario del Tesoro ${ }^{24}$. Para Estados Unidos era una cuestión de principio que no se restringiera el movimiento de sus inversionistas (efecto "precedente") y que el Central se "autolimitara" en la aplicación de controles cambiarios y financieros. También por principio, Chile no que- ría intervenir en las facultades del Central y en la práctica prefería mantener los grados de acción de la Autoridad Monetaria con estas herramientas ya probadas.

Como resultado final se optó por una formula "creativa", que salva ambas opciones. El Central reservó la mayor parte de sus atribuciones y podrá imponer restricciones a la entrada y salida de capitales por un año, sin tener que dar explicaciones ni compensar ${ }^{25}$. Incluso puede mantenerla por otro año, pero Estados Unidos tiene derecho a apelar a través del mecanismo de solución de controversias del Capítulo de Inversiones.

Es decir, en la práctica el Banco Central puede establecer un encaje hasta por dos años (recuérdese que en la década del 90 lo mantuvo por un año y diez meses). A partir del segundo año, un Panel puede determinar que la medida afecta "sustancialmente las transferencias" y que debe ser levantada ${ }^{26}$. En caso de sanciones o compensaciones, estas serán calculadas sin considerar el primer año y acotadas a las perdidas efectivas que la medida ocasionó.

\section{Conclusiones}

Chile tardó doce años en concretar un esperado Tratado de Libre Comercio con

${ }^{23}$ Paralelamente estaba el requisito de permanencia mínima de un año, que ya había eliminado el Banco Central, pero que sí mantenía el Estatuto de Inversiones Extranjeras, el DL 600 del Ministerio de Economía, y que se mantuvo.

${ }^{24}$ Un teórico prestigioso, conocido por "la regla de Taylor", una ecuación que describe los movimientos de una regla de política monetaria.

${ }^{25}$ Principalmente que no afecte la salida de utilidades, servicio de deuda, bonos u otros calendarizados por contrato.

${ }^{26}$ Las regulaciones de la Autoridad Monetaria se refieren al Mercado Formal, de modo que el impedimento sustancial a las transferencias es, a lo menos, rebatible. 
Estados Unidos. Mas no los desperdició. En ese período firmó acuerdos con toda Europa, sus vecinos latinoamericanos y Corea del Sur, reafirmando su liderazgo aperturista y su política de desarrollo basado en las exportaciones. Así, diversificó sus socios con trato preferencial y evitó aumentar la dependencia de ninguno en particular. En ese mismo tiempo creció impresionantemente y consolidó el menor riesgo país entre sus pares.

Con la misma paciencia deberá esperar los mismos doce años, pero esta vez para concretar la desaparición absoluta de aranceles, cuotas y otras barreras, que resultan del Tratado firmado con Estados Unidos. Dicho lapso sólo para un 4,5\% del comercio bilateral, netamente agrícola, porque al segundo año después de la firma ya no ha- brá aranceles industriales entre los países.

El impacto esperado es incierto en la magnitud, pero sin duda positivo. Junto a consolidar el actual nivel de exportaciones, la eliminación del escalonamiento arancelario y la certeza del arancel nulo, permitirá agregar valor a nuestras exportaciones y diversificar la canasta en el mediano plazo. El efecto sobre las inversiones puede ser aún mayor, ya que a partir de ahora cualquier empresa estadounidense que desee invertir en América Latina verá en Chile a un socio mucho más sólido y atractivo que cualquier otro.

Los logros son notables, queda en manos del sector empresarial aprovechar las oportunidades que el Gobierno ha abierto para ellos. 


\section{Anexos}

CuAdro $\mathrm{N}^{\circ} 1$. Acuerdos comerciales de Chile.

\begin{tabular}{|c|c|c|c|c|c|c|}
\hline \multicolumn{3}{|l|}{ Acuerdos } & \multicolumn{4}{|l|}{ Observaciones } \\
\hline \multicolumn{3}{|c|}{ APEC (Noviembre 1994) } & \multicolumn{4}{|c|}{ Asia Pacific Economic Cooperation. Acuerdo Regional en negociación. } \\
\hline \multicolumn{3}{|c|}{ ALCA (Diciembre 1994) } & \multicolumn{4}{|c|}{ Acuerdo de Libre Comercio de América. Acuerdo Regional en negociación. } \\
\hline \multicolumn{3}{|l|}{ OMC (Enero 1995) } & \multicolumn{4}{|c|}{ Ronda Uruguay de la OMC. Actualmente se negocia la Ronda Doha. } \\
\hline \multicolumn{7}{|c|}{ Acuerdos bilaterales en el marco ALADI } \\
\hline \multicolumn{2}{|l|}{ Acuerdos } & \multicolumn{2}{|l|}{ Vigencia } & \multirow{5}{*}{\multicolumn{3}{|c|}{$\begin{array}{l}\text { Acuerdos de Complementación Económica o de } \\
\text { "alcance parcial", que mantienen sectores excluidos. } \\
\text { Varios actualmente en profundización. } \\
\text { Chile adhirió a MERCOSUR como "asociado" y no como } \\
\text { miembro pleno. }\end{array}$}} \\
\hline \multicolumn{2}{|l|}{ México (Enero 1992) } & \multicolumn{2}{|c|}{ Ecuador (Enero 1995) } & & & \\
\hline \multicolumn{2}{|l|}{ Bolivia (Julio 1993) } & \multicolumn{2}{|c|}{ MERCOSUR (Octubre 1996) } & & & \\
\hline \multicolumn{2}{|c|}{ Venezuela (Julio 1993) } & \multicolumn{2}{|c|}{ Perú (Julio 1998) } & & & \\
\hline \multicolumn{2}{|c|}{ Colombia (Enero 1994) } & \multicolumn{2}{|c|}{ Argentina (Mayo 2000) } & & & \\
\hline \multicolumn{7}{|c|}{ Acuerdos de Libre Comercio } \\
\hline Acuerdo & \multicolumn{2}{|c|}{ Firma } & Vigencia & Acuerdo & Firma & Vigencia \\
\hline Canadá & \multicolumn{2}{|c|}{ Diciembre 1996} & Julio 1997 & Corea del Sur & Octubre 2002 & \multirow{3}{*}{$\begin{array}{l}\text { Acuerdos en trámite } \\
\text { Parlamentario. }\end{array}$} \\
\hline México & \multicolumn{2}{|c|}{ Abril 1998} & Agosto 1999 & Estados Unidos & Junio 2003 & \\
\hline Centroamérica (1) & \multicolumn{2}{|c|}{ Diciembre 1999} & Enero 2002 & EFTA (3) & Junio 2003 & \\
\hline Unión Europea (2) & \multicolumn{2}{|c|}{ Abril 2002} & Febrero 2003 & & & \\
\hline
\end{tabular}

\section{(1): Guatemala, Honduras, Nicaragua, Costa Rica y Salvador}

(2): Alemania, Austria, Bélgica, Dinamarca, España, Finlandia, Francia, Grecia, Holanda, Italia, Portugal, Reino Unido, Suecia, Luxemburgo e Irlanda. Se sumarán: Bulgaria, Letonia, Polonia, Lituania, Estonia, Rumania, República Checa, Eslovenia, Hungría y Eslovaquia.

(3): Suiza, Noruega, Islandia y Liechtenstein.

Fuente: DIRECON. 
Joseph Ramos y Alfie Ulloa Urrutia

Cuadro N². Algunos indicadores comerciales de los socios de EEUU.

\begin{tabular}{|c|c|c|c|c|c|c|c|c|}
\hline & 1993 & 1994 & 1995 & 1996 & 1997 & 1998 & 1999 & 2000 \\
\hline \multicolumn{9}{|c|}{ EEUU } \\
\hline$\%$ crecimiento PIB & 2.7 & 4.0 & 2.7 & 3.6 & 4.4 & 4.3 & 4.1 & 4.1 \\
\hline Total Importado (M) & 574.863 & 657.885 & 739.660 & 790.470 & 862.426 & 907.647 & 1.017 .435 & 1.205 .339 \\
\hline Total Exportado (X) & 439.295 & 481.887 & 546.464 & 582.137 & 643.222 & 634.705 & 642.189 & 712.287 \\
\hline \multicolumn{9}{|c|}{ México } \\
\hline$\%$ crecimiento PIB & 1.95 & 4.42 & -6.17 & 5.15 & 6.77 & 5.03 & 3.62 & 6.56 \\
\hline Arancel efectivo* & 2.1 & 1.4 & 0.8 & 0.6 & 0.6 & 0.5 & 0.5 & 0.2 \\
\hline Variación anual (X) & - & 25.7 & 27.0 & 20.2 & 14.6 & 9.4 & 17.2 & 23.6 \\
\hline$\%$ del total M por EEUU & 6.7 & 7.4 & 8.3 & 9.4 & 9.9 & 10.2 & 10.7 & 11.2 \\
\hline \multicolumn{9}{|c|}{ Canadá } \\
\hline$\%$ crecimiento PIB & 2.4 & 4.7 & 2.8 & 1.6 & 4.3 & 3.9 & 5.1 & 4.4 \\
\hline Arancel efectivo* & 0.4 & 0.3 & 0.2 & 0.2 & 0.1 & 0.1 & 0.1 & 0.0 \\
\hline Variación anual (X) & - & 7.8 & 12.5 & 7.9 & 7.4 & 4.1 & 13.5 & 15.5 \\
\hline$\%$ del total M por EEUU & 20.8 & 19.6 & 19.6 & 19.8 & 19.5 & 19.2 & 19.5 & 19.0 \\
\hline \multicolumn{9}{|c|}{ Chile } \\
\hline Tasa de crecimiento PIB & 7.1 & 5.7 & 10.6 & 7.5 & 6.6 & 3.2 & -0.8 & 4.2 \\
\hline Variación anual (X) & - & 24 & 11.6 & 10.1 & 3.9 & -2.8 & 16.2 & 1.9 \\
\hline$\%$ del total M por EEUU & 0.29 & 0.31 & 0.32 & 0.33 & 0.31 & 0.29 & 0.31 & 0.27 \\
\hline
\end{tabular}

* Aranceles efectivos pagados en Estados Unidos. Las cifras están en millones de dólares.

Fuente: Chomo 2002, Banco Central de Chile, Banco Central de México.

Cuadro N³. Exportaciones (X’s) E importaciones (M’s) de Chile.

\begin{tabular}{|c|c|c|c|c|c|c|c|c|c|c|}
\hline & \multicolumn{2}{|c}{ EEUU } & \multicolumn{2}{c|}{ MERCOSUR } & \multicolumn{2}{c|}{ UE } & \multicolumn{2}{c|}{ Asia } & \multicolumn{3}{c|}{ Total } \\
\hline & X's & M's & X's & M's & X's & M's & X's & M's & X's & M's \\
\hline 1990 & 1.490 & 1.373 & 642 & 1.124 & 3.351 & 1.893 & 2.305 & 965 & 8.610 & 7.742 \\
\hline 1991 & 1.595 & 1.582 & 785 & 1.332 & 2.976 & 1.562 & 2.711 & 1.317 & 9.068 & 8.207 \\
\hline 1992 & 1.655 & 1.985 & 982 & 1.740 & 3.067 & 2.027 & 3.221 & 1.869 & 10.116 & 10.129 \\
\hline 1993 & 1.656 & 2.477 & 1.087 & 1.761 & 2.557 & 2.313 & 2.976 & 2.047 & 9.431 & 11.134 \\
\hline 1994 & 2.059 & 2.638 & 1.365 & 2.054 & 2.941 & 2.396 & 3.992 & 2.134 & 11.960 & 11.820 \\
\hline 1995 & 2.330 & 3.793 & 1.765 & 2.677 & 4.275 & 3.155 & 5.502 & 2.655 & 16.027 & 15.900 \\
\hline 1996 & 2.592 & 4.110 & 1.748 & 2.814 & 3.761 & 3.538 & 5.287 & 2.840 & 15.546 & 17.824 \\
\hline 1997 & 2.699 & 4.333 & 1.843 & 3.193 & 4.037 & 3.958 & 5.682 & 3.129 & 16.654 & 19.662 \\
\hline 1998 & 2.625 & 4.026 & 1.662 & 3.130 & 4.213 & 3.850 & 4.086 & 3.074 & 15.077 & 18.779 \\
\hline 1999 & 3.133 & 3.025 & 1.531 & 3.095 & 4.232 & 2.850 & 4.727 & 2.389 & 16.257 & 15.144 \\
\hline 2000 & 3.195 & 3.338 & 1.709 & 4.338 & 4.548 & 2.880 & 5.687 & 2.950 & 18.466 & 18.089 \\
\hline Participación 90-00 & 17.0 & 21.2 & 10.3 & 17.7 & 27.1 & 19.7 & 31.4 & 16.4 & 100 & 100 \\
\hline Variación anual* & 8.2 & 10.8 & 11.1 & 15.3 & 4.3 & 5.8 & 11.2 & 13.3 & 8.7 & 9.8 \\
\hline
\end{tabular}

* Promedio simple

Fuente: Banco Central de Chile. 


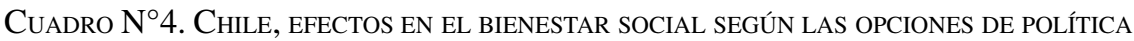
COMERCIAL.

\begin{tabular}{|l|c|c|c|c|c|c|}
\hline & MERCOSUR & NAFTA & $\begin{array}{c}\text { MERCOSUR } \\
\text { +NAFTA }\end{array}$ & $\begin{array}{c}\text { MERCOSUR } \\
\text { +NAFTA } \\
+ \text { +UE }\end{array}$ & $\begin{array}{c}\text { MERCOSUR } \\
\text { +Canadá } \\
\text { +México } \\
\text { +UE } \\
\text { +resto de } \\
\text { Sudamérica* }\end{array}$ & $\begin{array}{c}\text { MERCOSUR } \\
\text { +NAFTA } \\
\text { +UE } \\
\text { +resto de } \\
\text { Sudamérica }\end{array}$ \\
\hline Sin productos exceptuados & -0.43 & 1.04 & 1.48 & 5.24 & 8.16 & 8.4 \\
\hline Con productos exceptuados & -0.43 & 1.04 & 1.48 & 2.02 & 0.44 & 2.48 \\
\hline
\end{tabular}

* Sudamérica excluidos Chile y MERCOSUR.

Fuente: Harrison, Rutherford y Tarr.

Cuadro N5. Índice de Capítulos del TlC Chile-EEUU.

\begin{tabular}{|l|l|}
\hline I.- Provisiones Iniciales & XIII.- Telecomunicaciones \\
\hline II.- Definiciones Generales & XIV.- Entrada Temporal de Personas de Negocios \\
\hline $\begin{array}{l}\text { III.- Trato Nacional y Acceso a Mercado de } \\
\text { Bienes }\end{array}$ & XV.- Comercio Electrónico \\
\hline IV.- Reglas de Origen & XVI.- Políticas de Competencia \\
\hline V.- Administración Aduanera & XVII.- Propiedad Intelectual \\
\hline VI.- Medidas Sanitarias y Fitosanitarias & XVIII.- Asuntos Laborales \\
\hline VII.- Barreras Técnicas al Comercio & XIX.- Asuntos Ambientales \\
\hline VIII.- Defensa Comercial & XX.- Transparencia \\
\hline IX.- Contratación Pública & XXI.- Administración del Acuerdo \\
\hline X.- Inversiones & XXII.- Solución de Disputas \\
\hline XI.- Comercio Transfronterizo de Servicios & XXIII.- Excepciones \\
\hline XII.- Servicios Financieros & XXIV.- Provisiones Finales \\
\hline
\end{tabular}

Fuente: sitio Web del USTR.

Cuadro Nº6. Calendario de Desgravación para las exportaciones chilenas.

\begin{tabular}{|lcccccc|}
\hline Categoría & Totales & \multicolumn{2}{c}{ Agrícolas } & \multicolumn{2}{c|}{ Industriales } \\
\hline & US\$ & $\mathbf{\%}$ & US\$ & $\mathbf{\%}$ & US\$ & $\%$ \\
\hline Inmediata & 2.756 .482 & 87,0 & 865.309 & 84,0 & 1.891 .173 & 88,5 \\
\hline $\mathbf{2}$ años & 246.542 & 7,8 & - & - & 246.542 & 11,5 \\
\hline $\mathbf{4}$ años & 5.996 & 0,2 & 5.698 & 0,6 & 298 & 0,01 \\
\hline $\mathbf{8}$ años & 17.420 & 0,5 & 17.401 & 1,7 & 19 & 0,0 \\
\hline $\mathbf{1 0}$ años & 248 & 0,0 & 207 & 0,0 & 41 & 0,0 \\
\hline $\mathbf{1 2}$ años & 141.508 & 4,5 & 141.508 & 13,7 & - & - \\
\hline Total & $\mathbf{3 . 1 6 8 . 1 9 6}$ & $\mathbf{1 0 0}$ & $\mathbf{1 . 0 3 0 . 1 2 3}$ & $\mathbf{1 0 0}$ & $\mathbf{2 . 1 3 8 . 0 7 3}$ & $\mathbf{1 0 0}$ \\
\hline
\end{tabular}

Calendario aplicado a las cifras de exportación del 2001, en miles de dólares de Estados Unidos.

Fuente: DIRECON, Rosales (2003) 


\section{BIBLIOGRAFÍA}

Banco Central de Chile. (2001, febrero), "Comercio entre Chile y los Estados Unidos", Indicadores de Comercio Exterior.

Chomo, Grace. (2002). "Free Trade Agreements between developing and industrialized countries: comparing the US-Jordan FTA with Mexico's experience under NAFTA", US International Trade Commission Working Paper.

Frankel, JeFFrey; Romer, David. (1999). "Does trade cause growth?", The American Economic Review, vol. 89-3.

Harrison, Glenn; Rutherford, Thomas y Tarr, DAVID. (1997, mayo). "Trade Policy Options for Chile: a Quantitative Evaluation”. The North-South Agenda Papers.

Harrison, Glenn; Rutherford, Thomas; Tarr, DAVID. (1997). NAFTA, MERCOSUR and additive regionalism in Chile: A quantitative evaluation, Washington, D.C.

Harrison, Glenn; Rutherford, Thomas; Tarr, DAVID. (1999, abril). "Regional Trading Arrangements for Chile: Do the Results Differ with a Dynamic Model?", División del Comercio del Banco Mundial.

O.D.E.P.A. (2001, diciembre). "Inserción de la agricultura chilena en los mercados internacionales". Documento de Trabajo, ${ }^{\circ} 3$.
Larraín, Felipe; Coeymans, Juan E. (1994, diciembre). "Efectos de un Acuerdo de Libre Comercio entre Chile y Estados Unidos: un enfoque de equilibrio general", Cuadernos de Economía, no 94.

Meller, Patricio. (1998). Un siglo de economía política chilena (1890-1990), Santiago, Editorial Andrés Bello.

Rodrik, DANI. (2000, febrero), "Institutions for High-Quality Growth: GAT They are and How to Acquire Them", Working Paper, 7540, NBER.

(2000). "Trade Policy Reform as Institutional Reform”. BID, Diálogo Regional de Política, disponible también en http:// $\mathrm{k} \mathrm{s}$ g h o m e. h a r va r d.e d u / .drodrik.academic.ksg/Reform.PDF

Rosales, Osvaldo. (2003, abril). "El TLC ChileEstados Unidos”, presentación ante la Cámara Chileno Norteamericana de Comercio. Disponible en: www.direcon.cl/frame/acuerdos _internacionales/documentos/TLC-AMCHAMabril2003.PDF

SÁEZ, SEBASTIÁN. (1999). Estrategia y negociación en el sistema multilateral de comercio, Santiago, Ediciones Dolmen.

Sáez, Sebastián; Valdés, Juan Gabriel. (1999, abril). "Chile y su política comercial 'lateral"”, Revista de la CEPAL, n 67. 